INPLASY

PROTOCOL

To cite: Yang et al. Safety and Efficacy of Indocyanine Green Tracer-Guided Lymph Node

Dissection During Radical Gastrectomy. Inplasy protocol 202110085. doi:

10.37766/inplasy2021.1.0085

Received: 20 January 2021

Published: 21 January 2021

Corresponding author:

Jianqiao Yang

15069801810@163.com

Author Affiliation:

Department of

Gastroenterological Surgery,

Shandong Provincial Hospital

Support: None.

Review Stage at time of this submission: Data extraction.

Conflicts of interest:

None.

\section{Safety and Efficacy of Indocyanine Green Tracer-Guided Lymph Node Dissection During Radical Gastrectomy}

Yang, J1; Shang, L².

Review question / Objective: The meta-analysis was conducted to evaluate the effectiveness and safety of ICG fluorescence imaging-guided during radical gastrectomy. Condition being studied: Gastric cancer (GC) is the fourth most common cancer and the second most common cause of cancer deaths in the world, The treatment of gastric cancer has made great progress?However, even after curative resection, $40 \%-60 \%$ patients with advanced gastric cancer die of recurrence. This high early recurrence rate may be due to the unreliable preoperative diagnosis of lymph node status including micrometastasis.

INPLASY registration number: This protocol was registered with the International Platform of Registered Systematic Review and Meta-Analysis Protocols (INPLASY) on 21 January 2021 and was last updated on 21 January 2021 (registration number INPLASY202110085).

\section{INTRODUCTION}

Review question / Objective: The metaanalysis was conducted to evaluate the effectiveness and safety of ICG fluorescence imaging-guided during radical gastrectomy.
Condition being studied: Gastric cancer (GC) is the fourth most common cancer and the second most common cause of cancer deaths in the world, The treatment of gastric cancer has made great progress?However, even after curative 
resection, $40 \%-60 \%$ patients with advanced gastric cancer die of recurrence. This high early recurrence rate may be due to the unreliable preoperative diagnosis of lymph node status including micrometastasis.

\section{METHODS}

Participant or population: Inclusion criteria:patients of any sex, age, race or nationality who underwent gastrectomy for gastric cancer; exclusion criteria:) patients with a history of a drug?related allergy or of chemoradiotherapy, (2) patients previously treated with endoscopic mucosal resection (EMR) or endoscopic submucosaldissection (ESD).

Intervention: Using indocyanine green labeled near-infrared fluorescence laparoscopy underwent stomach tumor resection was the main intervention.

Comparator: The main comparator.

Study designs to be included: Either randomized controlled trials (RCTs), cohort studies, or comparative studies all will be included.

Eligibility criteria: The Cochrane Handbook was used to evaluate quality of randomized controlled studies (RCTs). If the study scored 4 or more out of a maximum of 6 points, it is considered as a high quality research and will be included. For nonrandomized controlled studies such as retrospective studies, the NewcastleOttawa scale was used to evaluate quality. The maximum score on the scale was 9 , and studies with scores $>6$ were considered to have high methodological quality and will be included.

Information sources: We will search the databases of Embase, PubMed, Web of Science, Cochrane Central Register of Controlled Trials, Chinese National Knowledge Infrastructure Database, Chinese Biomedical Literature Database, Chinese Science and Technique Journals
Database, and the Wan-fang Database for relevant papers.

Main outcome(s): Number of retrieved lymph node (including total lymph nodes harvested, total metastatic lymph nodes, number of D1 station lymph nodes, metastatic lymph nodes of D1 station, number of D2 station lymph nodes, metastatic lymph nodes of D2 station), duration of surgery, and postoperative complications rate.

Additional outcome(s): Intraoperative blood loss, postoperative first oral intake time, and postoperative hospital stay time.

Quality assessment / Risk of bias analysis: Potential publication bias will assessed byvisually inspecting the funnel plots based on primary outcomes. A study was considered to have no publication bias when the figure presented good symmetry. When possible, subgroup analyses of cancer staging, surgery type, type of primary tumor and resection, fibrosis grade, and imaging system used were performed to determine whether these factors affected the conclusion.

Strategy of data synthesis: The level of evidence was estimated by the Cochrane Evidence Centre in the UK .The Cochrane Review Manager software (RevMan; version 5.4) was used to calculate the pooled value of the odds ratios?OR) for categorical variables, mean difference (MD) for continuous variables, and the $95 \%$ confidence intervals (Cls) .A p 0.05 was considered statistically significant for all of the studys. The quantification of heterogeneity uses $\mathrm{I}^{2}$ measurement, and the $I^{2}$ values of $75 \%, 50 \%$ and $25 \%$ are considered as high, medium and low heterogeneity respectively.If high heterogeneity is observed, subgroup and sensitivity analyses are performed.If there is insignificant inter-study heterogeneity, fixed-effect models are used. If heterogeneity existed $(75 \%)$, the data were analyzed using a random-effects model. Funnel plots were created to identify potential publishing bias. 
Subgroup analysis: Subgroup analyses of cancer staging, surgery type, type of primary tumor and resection, fibrosis grade, and imaging system used were performed to determine whether these factors affected the conclusion.

Sensibility analysis: Sensitivity analysis was performed by removing one study at a time and repeating the meta-analysis to assess whether at least one study significantly affected the pooled estimates.

Language: None.

Country(ies) involved: China.

Keywords: indocyanine green, lymph node dissection, gastrectomy.

Contributions of each author:

Author 1 - Jianqiao Yang - The author drafted the manuscript.

Author 2 - Liang Shang.

Email: docshang@163.com 